Methods: A qualitative interview study was conducted. Participants were invited to use the website for two weeks and then participated in semi-structured interviews. The interview topic guide had been developed in line with current literature. The interviews were audio recorded and transcribed verbatim. Thematic analysis was used by the main researcher (with independent verification) to interrogate the data and identify themes.

Results: Six people with low health literacy (S-TOFHLA 17 \pm 3 ) were recruited from community groups from an inner-city area with a high index for social deprivation. Four key themes were identified. 1) Dealing with technical issues, where participants demonstrated that they could persevere with technical problems in using online resources; 2) Information overprovision, here participants found there was too much complex health information provided, that hindered usability; 3 ) Motivation for information seeking, where participants discussed that it was pain that encouraged resource use but motivation to self-manage was influenced by personal beliefs; 4) Specific professional input, the participants whilst willing to use and engage with the website recalled that they also want to have access to a health professional and felt the website could supplement but not replace this contact. "I think you have it from the health care where the healthcare are telling you and explaining to you".

Conclusions: Digital online OA self-management presented by MyJointPain showed potential for use by people with joint pain and lower health literacy levels. This is important as outcomes for this group are poor (1). Text volume, detail and image use should be carefully considered when designing new online resources and involvement of people with lower health literacy when designing online tools will help to ensure information is accessible and useful to all OA patients. Clinicians should consider their patients' health literacy level, computer literacy and readiness to change behaviour before prescribing web based self-management tools.

References:

[1] Luoung et al (2012) Aging health. 8(4): 413-437. doi:10.2217/ahe.12.43.

[2] Wallace $L$ (2006) Annals of Family Medicine 4:85-86.

[3] Adams J et al (2016) Rheumatology 55 (S1):i8-i9.

Acknowledgements: Prof D. Hunter, A. Cahill and C. Dickson from Arthritis Australia.

Disclosure of Interest: None declared

DOI: 10.1136/annrheumdis-2017-eular.1529

\section{SAT0763-HPR FUNCTIONAL OUTCOMES IN PATIENTS WITH RHEUMATOID ARTHRITIS ON THE BACKGROUND CORRECTION OF PROGESTERONE INSUFFICIENCY}

M. Salokhiddinov ${ }^{1}$, H. Ahmedov ${ }^{2} .{ }^{1}$ Tashkent Medical Academy; ${ }^{2}$ General practicioner, Tashkent Medical Academy, Tashkent, Uzbekistan

Background: It is known that one of the main demands of the health system is to improve preventive measures. In particular, issues of optimizing secondary prevention are very important, as it gives an opportunity to reduce the progression of the disease and complications, as well as improve the quality of life of patients. Therefore, the study values the correction of progesterone deficiency on the background of preventive and rehabilitation interventions in a general medical practice in patients with RA is of practical interest.

Objectives: Studying the role of progesterone deficiency correction efficiency to optimize secondary prevention in patients with RA in a general medical practice. Methods: Study involved 180 RA patients aged $35,6 \pm 3,9$ years. The study group (group 1) consisted of 80 patients with RA, the second 50 (group 2) and third and 50 (group 3). 1 group was carried preventive and curative interventions, based on an integrated approach with the inclusion of preventive and rehabilitation interventions on the background correction of progesterone deficiency (Duphaston) in a general medical practice. Group 2 is the same, but without the correction of progesterone deficiency, and the third group only took pathogenetic treatment. Group 3 as opposed to the 1 and 2 had displays of progesterone deficiency. For an objective assessment of the effectiveness of using EULAR recommendations, assessing the improvement in \%, according to the ACR, and to assess the functional status - health status index questionnaire Stanford Health Assessment Questionnaire (HAQ)

Results: The impact of interventions carried out by us on the functional outcome showed some differences between groups. Thus, according to ACR 50 criteria dynamics between groups were significant difference at 24 months, since improvement was noted in $52 \%$ of group 1 and $32 \%$ in group $2(p=0.032)$. By the end of 3 months of intervention minimal effect on performance within the $\mathrm{HAQ} 0,22 \leq \triangle \mathrm{NAQ} \leq 0,36$ was observed in $80 \%$ of group $1,78 \%$ in 2 nd group and $76 \%$ in group $3(p>0.05)$, while not a single case was fixed not satisfactory or pronounced effect. By the end of 12 months, the dynamics of functional status was noticeable and patients in group 1 have prevailed a satisfactory effect within $0,36 \leq \triangle H A Q<0.80-48.75 \%(p=0.043$ and $p=0.048$, respectively), so a marked effect within $\triangle H A Q \geq 0,80$ functional status - $15 \% \quad(p=0.001 ; p=0.011$, respectively). This trend continued and by the end of 24 and 36 months, as the group 1 compared with group 2 is almost 8 times $(p=0.001)$ and group 32 times (p. $=0.045$ ) greater $\triangle H A Q \geq 0,80$ a marked effect.

Conclusions: The preventive and therapeutic interventions on the background correction of progesterone deficiency in RA patients in a general practice reliably delivers stable ACR 50 response (64\%) and significantly improves the functionality of the joints in RA.
References:

[1] Akunts K.B. Menopause. M., Triada-H, 2004.

[2] Nasonov E.L. Rheumatology. Clinical recommendations. Moscow: GEOTARMedia; 2010.

[1] Smolen J.S. Aletaha D, Bijlsma J.W, et.al. Treating rheumatoid arthritis to target: recommendations of an international task force. Ann Rheum Dis. 2010.

Disclosure of Interest: None declared

DOI: 10.1136/annrheumdis-2017-eular.1067

\section{SAT0764-HPR RETROSPECTIVE STUDY ABOUT ASSOCIATION OF MENTAL AND SLEEP DISORDERS AMONG ADULTS WITH PAINFUL MUSCULOSKELETAL CONDITIONS}

S. Abdul Latif ${ }^{1}$, A. Abdel Zaher ${ }^{2}$, K. Al Hassanein ${ }^{3}$, M. Zaghloul ${ }^{1}$, M.H. Salama ${ }^{4}$, E. Barakat ${ }^{1}$, E. Mohamed ${ }^{3}$, A. Shaky ${ }^{3} .{ }^{1}$ Rheumatology, Saudi German hospital, Jeddah; ${ }^{2}$ Rheumatology, Saudi German hospital, Medinah; ${ }^{3}$ Rheumatology, Saudi German hospital, Riyadh, Saudi Arabia; ${ }^{4}$ Rheumatology, Thumby Hospital, Dubai, United Arab Emirates

Background: Chronic pain is a common condition that affects one-third of the population, accounting for a large number of medical consultations and a significant proportion of health care costs.

Evidence suggests that anxiety and depression are associated with increased pain sensitivity and pain-related disability, co-morbid states that are more disabling than either condition alone.

Likewise, sleep disorders have also been linked with chronic pain, and people who experience pain-related sleep disturbances are significantly more disabled than those who do not.

Objectives: The objective of this study was to determine the prevalence of anxiety, depression, and sleep disorders, painful musculoskeletal conditions in a sample of adults with disabilities.

Methods: This cross-sectional study analyzed data of 1692 adults aged 18 or older who have disabilities over 10 years (2005 to 2014). We examined the following chronic conditions, in which pain is a key symptom and forms part of the diagnosis, and organized them into 3 groups. Group 1 consisted of (rheumatic diseases), arthritis, osteoarthritis, rheumatoid arthritis, and ankylosing spondylitis; group 2 consisted of muscular dystrophy; and group 3 consisted of neck or back pain;

The prevalence (95\% confidence interval) of painful musculoskeletal conditions was determined according to the diagnosis. Factors associated with these painful conditions were analyzed separately for men and women by using a logistic regression model.

Results: The prevalence of painful musculoskeletal conditions was $66.9 \%$ (95\% $\mathrm{Cl}, 66 \%-67.5 \%)$. Factors associated with these conditions in both men and women included older age, a sleep disorder, and concomitant chronic anxiety and/or depression was $23.5 \%(95 \% \mathrm{Cl}, 22.5 \%-24 \%)$, all of which were higher in women than in men. Of the 1692 adults with disability included in the study, $65 \%$ were women. The average age was 50.5 (standard deviation, $12.5 \mathrm{y}$ ), and $65 \%$ of participants were 50.5 or older. In addition, $48 \%$ of participants were married. Of the participants, $16 \%$ had been diagnosed with chronic anxiety and $22 \%$ with chronic depression; and $32 \%$ with sleep disorder (sleeping 6 hours or less per day. Prevalence of Painful Conditions in Adults with Disabilities, by Group of Conditions and by Sex, Differences between men and women were significant at $\mathrm{P}<.001$ (for all groups of conditions, except muscular dystrophy.

Conclusions: The prevalence of painful musculoskeletal conditions associated with depression, anxiety, and/or a sleep disorder, is high in people with disability, especially in women. To design plan for rehabilitation and improving the quality of life of adults with disability and painful conditions; treatments for depression, anxiety and/or sleep disorders should be considered in addition to conventional treatments.

Disclosure of Interest: None declared

DOI: 10.1136/annrheumdis-2017-eular.3353

\section{SAT0765-HPR CRITERION AND CONCURRENT VALIDITY OF THE AMERICAN-ENGLISH VERSION OF THE FLARE QUESTIONNAIRE TO DETECT AND MEASURE FLARES IN RHEUMATOID ARTHRITIS}

N. Barroso ${ }^{1}$, T.G. Woodworth ${ }^{1}$, F. Guillemin ${ }^{2}$, D.E. Furst ${ }^{1}$, J. Brook ${ }^{1}$, S. Kafaja ${ }^{1}$ N. Borazan ${ }^{1}$, D.A. Elashoff ${ }^{1}$, B.J. Fautrel ${ }^{3}$, V.K. Ranganath ${ }^{1}$. ${ }^{1}$ Medicine, UCLA, Los Angeles, United States; ${ }^{2}$ Université de Lorraine, Paris Descartes University, APEMAC, EA 4360; ${ }^{3}$ Pitié Salpêtrière Hospital, Paris, France

Background: Despite advances in rheumatoid arthritis (RA) therapeutic agents, difficulty in managing RA flares persists, and remission or the persistent absence of inflammation remains challenging to achieve. The French FLARE (F-FLARE) was developed to facilitate and standardize detection and measurement of RA flares between clinic visits. ${ }^{1,2}$ We have previously reported the cognitive debrief for the published British English version, which determined that revision of wording and presentation of items was required. Here we report validation of the American English version of FLARE (Am-E FLARE-RA). ${ }^{3}$

Objectives: To assess whether the Am-E FLARE questionnaire performs comparably to the French version of FLARE in detecting RA flare in an American English-speaking clinic population. 
Methods: We enrolled patients attending UCLA rheumatology clinics. After informed consent, patients completed a questionnaire to collect demographics, Am-E FLARE, RAPID3, patient global visual analog scale (VAS), and self-reported flare at time of visit or between visits (yes/no). Questionnaires were provided to patients without specific instruction. From the electronic medical records, we extracted MD global VAS, MD-reported flare, seropositivity, disease duration, swollen/tender joint counts (SJC/TJC), and calculated the clinical disease activity index (CDAI). Analyses included Wilcoxon rank sum tests and Spearman correlations to assess criterion and concurrent construct validity.

Results: Eighty-five RA patients diagnosed by the1987 American College of Rheumatology (ACR) criteria enrolled in our study. For the study population as a whole, mean age was about 50 years and most were female. Mean disease duration was about 10 years and about $65 \%$ were seropositive. (see Table). Am-E FLARE scores were significantly higher in patients' self-reporting flare compared to patients who reported no flare $(\mathrm{p}=0.005)$, and also for patients with MD-reported flare compared to those without flare $(p=0.008)$ (see Table). Interestingly, there were no significant differences in SJC $(p=0.82)$ and physician global $(p=0.19)$ between patients who self-reported flare versus those who reported no flare. In addition, the Am-E FLARE scores correlated moderately with both CDAI (corr=0.46) and RAPID3 (corr=0.57).

\begin{tabular}{|c|c|c|c|c|c|c|}
\hline & \multicolumn{2}{|c|}{ Patient-reported FLARE } & \multicolumn{4}{|c|}{ MD-reported FLARE } \\
\hline & No Flare & Flare & & No Flare & Flare & \\
\hline & $\begin{array}{c}\text { Mean (SD)/ } \\
\begin{array}{c}N(\%) \\
n=51\end{array}\end{array}$ & $\begin{array}{c}\text { Mean (SD)/ } \\
N(\%) \\
n=34\end{array}$ & ${ }^{*}$ p-value & $\begin{array}{c}\text { Mean (SD)/ } \\
\begin{array}{c}N(\%) \\
\mathbf{n}=67\end{array}\end{array}$ & $\begin{array}{c}\text { Mean (SD)/ N } \\
(\%) \\
n=18\end{array}$ & ${ }^{*}$ p-valu \\
\hline Age & $52.37(15.62)$ & $47.15(16.29)$ & 0.15 & $51.73(16.09)$ & $44.89(14.87)$ & 0.08 \\
\hline Female & $46(90.20)$ & $31(91.18)$ & 0.88 & $60(89.55)$ & $17(94.44)$ & 0.53 \\
\hline Race $^{*}$ & $11(21.57$ & $5(14.71)$ & & $16(23$ & $0(0,00)$ & \\
\hline $\begin{array}{l}\text { Asian } \\
\text { Black }\end{array}$ & $\begin{array}{l}5(9.80) \\
\end{array}$ & $5(14.71)$ & & $8(11.94)$ & $2(11.11)$ & \\
\hline Pac Island & $1(1.96)$ & $1(2.94)$ & 0.79 & $1(1.49)$ & $1(5.56)$ & 0.22 \\
\hline Caucasian & $32(62.75)$ & $23(67.65)$ & & $40(59.70)$ & $15(83.33)$ & \\
\hline Other & $2(3.92)$ & $0(0.00)$ & & $2(2.98)$ & $0(0.00)$ & \\
\hline Seropositivity ${ }^{*}$ & $32(62.75)$ & $23(67.65)$ & 0.64 & $42(62.69)$ & $13(72.22)$ & 0.45 \\
\hline Disease duration & $9.63(9.07)$ & $11.88(10.98)$ & 0.41 & $11.06(10.21)$ & $8.56(8.50)$ & 0.32 \\
\hline RAPID3 & $9.24(6.23)$ & $14.64(5.79)$ & 0.0001 & $9.67(6.03)$ & $17.63(4.38)$ & $<.0001$ \\
\hline CDAI & $17.49(13.48)$ & $22.88(13.03)$ & 0.03 & $16.74(11.54)$ & $30.11(15.19)$ & 0.0003 \\
\hline TJC & $6.29(6.67)$ & $8.91(7.24)$ & 0.04 & $6.25(6.36)$ & $11.39(7.85)$ & 0.005 \\
\hline SJC & $4.67(4.94)$ & $4.56(3.96)$ & 0.82 & $3.96(3.88)$ & $7.11(5.94)$ & 0.005 \\
\hline Patient Global & $3.33(2.39)$ & $5.15(2.33)$ & 0.001 & $3.45(2.32)$ & $6.22(2.02)$ & $<.0001$ \\
\hline Physician Global & $3.20(2.08)$ & $3.85(2.24)$ & 0.19 & $2.94(1.92)$ & $5.39(1.91)$ & $<.0001$ \\
\hline FLARE Total & $4.80(3.32)$ & $6.88(2.01)$ & 0.005 & $5.18(3.07)$ & $7.31(2.27)$ & 0.008 \\
\hline FLARE Physical & $5.11(3.47)$ & $7.28(2.02)$ & 0.008 & $5.49(3.21)$ & $7.81(2.10)$ & 0.005 \\
\hline FLARE Emotional & $4.48(3.47)$ & $6.49(2.61)$ & 0.007 & $4.88(3.33)$ & $6.81(2.71)$ & 0.03 \\
\hline
\end{tabular}

Joint Count; SIC = Swollen Joint Count; Seropositivity = either CCP + or RF+; *Wilcoxon rank sum test; \#Race and Seropositivity are presented as frequencies and present and p-values are calculated using Chi-square test

Conclusions: The Am-E FLARE is feasible for use in clinic, and shows good criterion validity, with scores significantly higher in patients who self-report flare. In addition, Am-E FLARE shows good discriminant validity, distinguishing patients who are flaring according to MD or patient self-report from those who are not. Finally, Am-E FLARE demonstrates construct validity comparable to that of the original French version of FLARE.

\section{References:}

[1] Jean-Marie Berthelot, Michel De Bandt, Jacques Morel, et al. A tool to identify recent or present rheumatoid arthritis flare from both patient and physician perspectives: The "FLARE" instrument. Ann Rheum Dis 2012;71:1110-1116.

[2] Fautrel B, Morel J, Berthelot JM, et al. Validation of FLARE-RA, a selfadministered tool to detect recent or current rheumatoid arthritis flare. Arthritis Rheumatol. 2016 Sep. 16

[3] EULAR14-SCIE-4820.

Disclosure of Interest: None declared

DOI: 10.1136/annrheumdis-2017-eular.3329

\section{SAT0766-HPR THE FACTORS AFFECTING FUNCTIONAL ABILITIES IN PATIENTS WITH JUVENILE IDIOPATHIC ARTHRITIS}

E. Tarakci ${ }^{1}$, N. Arman ${ }^{1}$, O. Kasapcopur ${ }^{2}$. ${ }^{1}$ Division of Physiotherapy and Rehabilitation, Faculty of Health Science; ${ }^{2}$ Department of Pediatric Rheumatology, Medical Faculty of Cerrahpasa, Istanbul University, Istanbil, Turkey

Background: Juvenile idiopathic arthritis $(\mathrm{JIA})$ is the most common rheumatic disease in pediatric population and is characterized by a heterogeneous group of diseases that include chronic arthritis of unknown origin, which begins before 16 years of age (1). Patients with JIA commonly experience acute and chronic pain, decreased mobility, and joint stiffness leading to restrictions on functional ability and isolation from their peers (2).

Objectives: The aim of this study was to assess relationship between clinical features and functional ability and determine the factors affecting functional abilities in patients with JIA

Methods: 172 (aged 5-18 years) with JIA were included in this study. Sociodemographic data, clinical features (duration of disease, subtype of JIA, and number of involved joint) and functional ability were assessed with Childhood Health Assessment Questionnaire (CHAQ). Pain and overall well-being were measured using a Visual Analog Scale (VAS). Relations between the factors affecting functional abilities in JIA were assessed by multiple linear regression analysis

Results: The mean age was $10.71 \pm 3.47$ and the mean disease duration was $5.41 \pm 3.53$ years. Score of $C H A Q$ was affected by number of involved joint $(\beta=0.18$, $p<0.05)$, subtypes of JIA $(\beta=-2.75, p<0.01)$ and pain $(\beta=0.15, p<0.05)$, but were not affected by age $(\beta=-0.03, p>0.05)$, gender $(\beta=-0.03, p>0.05)$ and disease duration $(\beta=0.03, p>0.05)$. In addition to, significant difference was found between all CHAQ subscores (except of "walking") of polyarticular and oligoarticular JIA $(p<0.001)$. Functional abilities of patients with polyarticular JIA were lower than functional abilities of patients with oligoarticular JIA.

Conclusions: In the literature, it reported that functional activities were adversely affected in patients with JIA, when they compared with healthy subjects. In our study, we found that functional abilities were affected by subtypes of JIA, number of involved joint, and pain. But, these were not affected by age, gender, and disease duration in JIA. We suggest that subtypes of JIA, number of involved joint, and pain should be considered, when patients with JIA are directed to physical therapy program to encourage functional abilities.

References:

[1] Prakken B, Albani S, Martini A (2011) Juvenile idiopathic arthritis. Lancet 377:2138-2149.

[2] Tarakci, E., Yeldan, I., Mutlu, E. K., Baydogan, S. N., \& Kasapcopur, O. (2011). The relationship between physical activity level, anxiety, depression, and functional ability in children and adolescents with juvenile idiopathic arthritis. Clinical rheumatology, 30(11), 1415

Disclosure of Interest: None declared

DOI: 10.1136/annrheumdis-2017-eular.4923

\section{SAT0767-HPR PHYSICAL ACTIVITY MAINTENANCE AND DIGITAL HEALTH INTERVENTIONS IN PEOPLE WITH RHEUMATOID ARTHRITIS: PATIENT AND HEALTHCARE PROFESSIONALS' PERSPECTIVES}

R. Simmons ${ }^{1}$, H. Lempp ${ }^{2}$, J. Galloway ${ }^{2}$, L. Bearne ${ }^{1}$. ${ }^{1}$ Division of Health and Social Care Research, Kings College London University; ${ }^{2}$ The Academic Department of Rheumatology, Kings College London, London, United Kingdom

Background: Physical Activity (PA) improves health and function in people with Rheumatoid Arthritis (RA) but people with RA commonly fail to meet recommended PA levels. Digital Health Interventions (DHIs) are delivered through digital media to facilitate behaviour change, such as maintaining PA. To date, little is known about the perceptions of people who have RA or their healthcare professionals (HCPs) about the factors that influence PA and the desirability and perceived suitability of using DHI to support PA maintenance.

Objectives: To explore RA patients' and HCPs' perceptions of the factors that influence PA maintenance as well as their views on the use of DHIs to support sustained PA.

Methods: Adults aged $>18$ years with established RA were recruited from two inner-city NHS hospitals. Semi-structured interviews, using a topic guide developed a priori, were conducted by one researcher either face-to-face or by telephone until data saturation of themes was reached. HCPs working at one NHS inner-city hospital, who self-reported $>3$ months experience managing RA patients attended one semi-structured focus group, facilitated by two researchers and informed by a topic guide.

The interviews and focus group were audio-recorded, transcribed verbatim and the data analysed thematically. Themes were corroborated by a sample of participants and an independent researcher.

Results: 11 patients were recruited between January-May 2016 (five females; mean age 58 years [standard deviation 17 years]). Five themes were identified from the interviews: 1) Balancing the benefits of activity with the costs; 2) Gaining knowledge about safe and effective PA; 3) Disease control; 4) The environment matters; and 5) Interaction of person, disease and DHI.

Patient participants were largely aware of the benefits of PA but wanted more information about safe PA. Ongoing PA was informed by participants balancing the benefits of PA with concerns about aggravating symptoms. Good symptoms control facilitated PA but severe and unpredictable symptoms and unsuitability of exercise facilities were perceived as barriers to PA maintenance. Patient participants mostly stated that DHIs were a desirable and acceptable adjunct to supporting PA maintenance.

The focus group was conducted with six HCPs (two physiotherapists, two rheumatologists, one podiatrist and one medical student). Two themes were identified 1) Inactivity demands a proactive response by HCPs and 2) DHIs have potential to support PA, but "there is no panacea".

HCP participants universally agreed that PA was beneficial and that it was important to support ongoing PA through education and multimodal interventions, including DHIs.

Conclusions: Disease control, knowledge and environmental factors influence PA maintenance in people with RA, who balance the perceived benefits with the potential costs of PA. HCPs recognise the need to proactively support PA maintenance. DHIs offer a potentially useful and acceptable tool to support PA maintenance.

Disclosure of Interest: R. Simmons Grant/research support from: NIHR MRes fellowship, H. Lempp: None declared, J. Galloway: None declared, L. Bearne: None declared 